\title{
Food Physical Processing Technology and its Basic Framework
}

\author{
Dong Tianfei \\ Chifeng Industrial Vocational Technical College, Chifeng City, Inner Mongolian Autonomous Region, China, 024000
}

\begin{abstract}
During the development of traditional food processing technology, there are great challenges in actual processing efficiency and product quality, which also makes the application of modern physical technology one of the effective measures to solve the above challenges. To this end, the relevant departments and staff need to strengthen the research emphasis on food physical processing technology. This article summarizes the problems faced by my country's food industry based on previous work experience, and discusses the basic framework of food physical processing technology from two aspects of physical methods and food processing methods.
\end{abstract}

\section{Introduction}

The food industry covers a very wide range, such as food raw material production, processing and manufacturing, and consumption. It has a direct relationship with many links in the industrial chain, and is also the key content of my country's national economic development. However, it can be learned from the traditional food processing link that there are problems such as low raw material utilization rate and poor processing efficiency, which has greatly hindered the development of the food industry. Practical research shows that the introduction of physical methods is of great significance for solving traditional food processing problems and can maintain the leap-forward development of the food industry.

\section{Problems Facing Our Country's Food Industry}

At this stage, my country is in a critical period of economic development transformation, from a middle-income to a high-income country, and the overall national consumption structure is constantly changing. In this new normal economic field, many new requirements are put forward for the structural adjustment and transformation and upgrading of the actual food industry.

\subsection{Obvious Structural Problems}

In general, my country's food industry chain is incomplete, which also makes raw material production, warehousing and logistics and other aspects not constitute a good whole, let alone establish internal links. my country's actual product structure continues to be optimized, and the proportion of actual deep-processed products has fallen sharply in recent years. In addition, my country's food industry does not possess strong international competitiveness. Although the absolute value of our country's food exports is on the rise, the proportion of actual exports has declined significantly, from $5.44 \%$ in 2010 to $2.62 \%$ in 2018 . More importantly, the energy consumption and resource consumption of products in my country's food industry are extremely high, which also leads to many deficiencies in green and low-carbon processing and green manufacturing technology development.

\subsection{Food Industry System Lags Behind}

Throughout the development of the entire food industry in China, the actual raw material production is mainly based on the agricultural industry, combined with food processing and food logistics service industries to build a food quality and safety monitoring system. In this process, there are many industries involved, such as primary industry, secondary industry and tertiary industry, which are both interconnected and independent. For the high-end value points in the industrial system, the main content is concentrated in sales, cooking and other links. However, my country's main force in food processing is scattered among farms and factories in various regions, engaged in terminal cooking and sales. The major industry leaders themselves do not have strong innovation capabilities, which also leads to the slow development of the food industry's own equipment manufacturing and logistics distribution, which cannot keep up with the needs of the times.

\subsection{The Value Chain Concept Parallel to the Food Industry Chain Needs to be Strengthened}

In contrast, our country's food industry innovation is not closely integrated with the market, coupled with the "farming and pig raising" and " the processing manufacturing" models, our country's agricultural and 
industrial transformation has been greatly constrained. In addition, there is not much research on brands, marketing, and investment by manufacturers, which makes existing foods unable to meet people's growing nutrition and health requirements. Therefore, in the transformation and development of the actual food industry, my country needs to change from the low-end to the high-end in the value chain. At this stage, my country has entered a period of intensive innovation and technological reforms are becoming more and more perfect. At the same time, my country's economy and society have entered a stage of transformation and upgrading. To this end, relevant departments need to focus their research on problem solving in the food industry and develop reasonable innovation drivers. Only after scientific and technological innovation is achieved can new opportunities be created for processing and manufacturing, and the gradual development of the food industry can be maintained.

\section{Food Physical Technology}

\subsection{Application of Physical Methods in Degradation and Modification of Biological Macromolecules}

Ultrasound or microwave should be used to explain the modification and other reactions aimed at the extraction of biological macromolecules in natural products. While strengthening the reaction rate, avoid the application of organic solvents to ensure that the food is always in a safe state. First of all, in the preparation of peptide ultrasonic enzymes, the actual ultrasonic has been applied to the enzymatic hydrolysis reaction from three aspects, such as catalyst protease ultrasonic pretreatment. In addition, ultrasound will also have a positive effect on substrate protein pretreatment. At the same time, ultrasound-assisted enzymes must be used to achieve enzymatic hydrolysis in ultrasound of the appropriate intensity, which is also the essence of strengthening protease activity. Secondly, the earliest research object in the process of ultrasonic degradation modification of polysaccharides is organic pollutants, which is actually the study of polysaccharide degradation. The earliest research can be traced back to the 1930s. The main degradation indicators of this type of research include viscosity and molecular mass distribution. From a structural and functional point of view, heteropolysaccharides have a special molecular structure, and researchers can use ultrasound to achieve a comprehensive study of licorice polysaccharides and yam polysaccharides. In general, the structure-activity relationship of polysaccharides is directly related to the molecular weight and antithrombotic activity of polysaccharides. In order to better present the structure of polysaccharides, one can conduct in-depth research on the polysaccharide molecules of laver to understand the specific degradation process of polysaccharide molecules.

\subsection{Application of Physical Methods in Food Microbial Fermentation}

It can be seen in the microbial physical mutagenesis that the actual mutation breeding can be effectively treated according to different types of physical mutagenesis and chemical mutagenesis. Among them, physical mutagenesis can show better safety and effect during use, so that subsequent applications become more extensive. Common physical mutagenesis factors are ultraviolet, X-ray, fast neutron and laser. Among them, the application is frequent as ultraviolet rays, and the actual mutagenesis effect is excellent. In addition, with the help of physical forms such as magnetism and laser, microbial strains can be changed under extreme conditions to increase the yield of target substances during fermentation. In addition, for the application of ultrasound-assisted fermentation technology, it is necessary to effectively stimulate the bacterial activity under the action of a weak magnetic field or ultrasonic waves, which is also the fundamental reason for enhancing the fermentation efficiency. Researchers Yu Shujuan and others carried out soy sauce production research experiments with the help of ultrasonic catalyzed fermentation of Aspergillus oryzae. Under the action of $40 \mathrm{kHz}$ and $10 \mathrm{~W}$ ultrasound, the fermentation rate of soy sauce can be $150 \%$ higher than the previous traditional process, and the actual aspergillus oryzae spore growth rate can be Increase by more than $30 \%$. Through the microscope, we can observe that the aspergillus oryzae cells under the action of ultrasound will not easily break.

\subsection{Food Physics Non-thermal Processing Technology}

With the continuous improvement of people's living standards, consumers are more inclined to natural flavored foods during the consumption process, and they have new requirements for freshness and nutritional value, which also makes micro-processed foods occupy a place in the market. In order to promote the development of this type of technology, many countries have begun to promote the physical non-thermal sterilization technology of food, such as ultra-high voltage, pulsed electric field and so on. In general, physical non-thermal sterilization has become one of the most concerned in the field of international food technology. Through research, Ma Haile and others found that if the magnetic field strength is higher, the sterilization effect will become lower and lower, and will not increase monotonously with the increase of the magnetic field strength. In addition, the researcher also used the study of calcium ion transmembrane behavior in the system to confirm that high-intensity pulses have a great effect on the permeability of microbial cell membranes. Therefore, in the actual high-intensity pulsed magnetic field sterilization, it is of positive significance to change the permeability of the cell membrane. More importantly, it can also be seen from the transmembrane behavior of calcium ions in microbial cells that there is a close relationship with the actual biological window effect. 


\subsection{Optimizing Innovative Deployment of Food Physical Processing}

In the implementation of this work, the most important thing is to strengthen basic research. Staff should focus on the mechanism of sterilization and inactivation of enzymes in food physical processing technology, and conduct in-depth research on subsequent food quality and safety issues. Secondly, based on cutting-edge technology research, the entire food physical processing engineering technology is more scientific and rational. Last but not least, it focuses on the development and research of common key technologies and engineering equipment. If you want to truly upgrade the food specialty, you should take the breakthrough of intelligent equipment as the basis to ensure that the key technologies of physical processing are effectively developed and utilized. Finally, strengthen the transformation of scientific and technological achievements, take collaborative innovation as the basis, accelerate the research and commercial application of physical processing technology, and do a good job in personnel training to truly achieve a reasonable deployment of the food physical processing innovation process.

\section{Basic Framework of Food Physical Processing Technology}

\subsection{Physical Method}

Due to the large differences between the properties of the physical field, the actual food physical processing technology mainly involves two situations, namely non-thermal physical processing, thermal physical processing and physical rapid detection, as shown in Figure 1. Among them, non-thermal physical processing mainly uses physical means. In recent years, the related research theories have gradually increased, which has also played a positive role in the industrialization of the entire food industry. In addition, many countries have also begun to study the relationship between non-thermal physical processing technology and enzymatic hydrolysis reaction, so that they have a new understanding in the process of modification and modification of biological macromolecules, and the development potential is huge. Due to the continuous advancement of physical methods, rapid detection technology for food quality has been comprehensively developed, especially in basic theoretical research and industrialization reactions, which has made great breakthroughs. This is also an important development direction for subsequent quality inspection to process parameter detection.

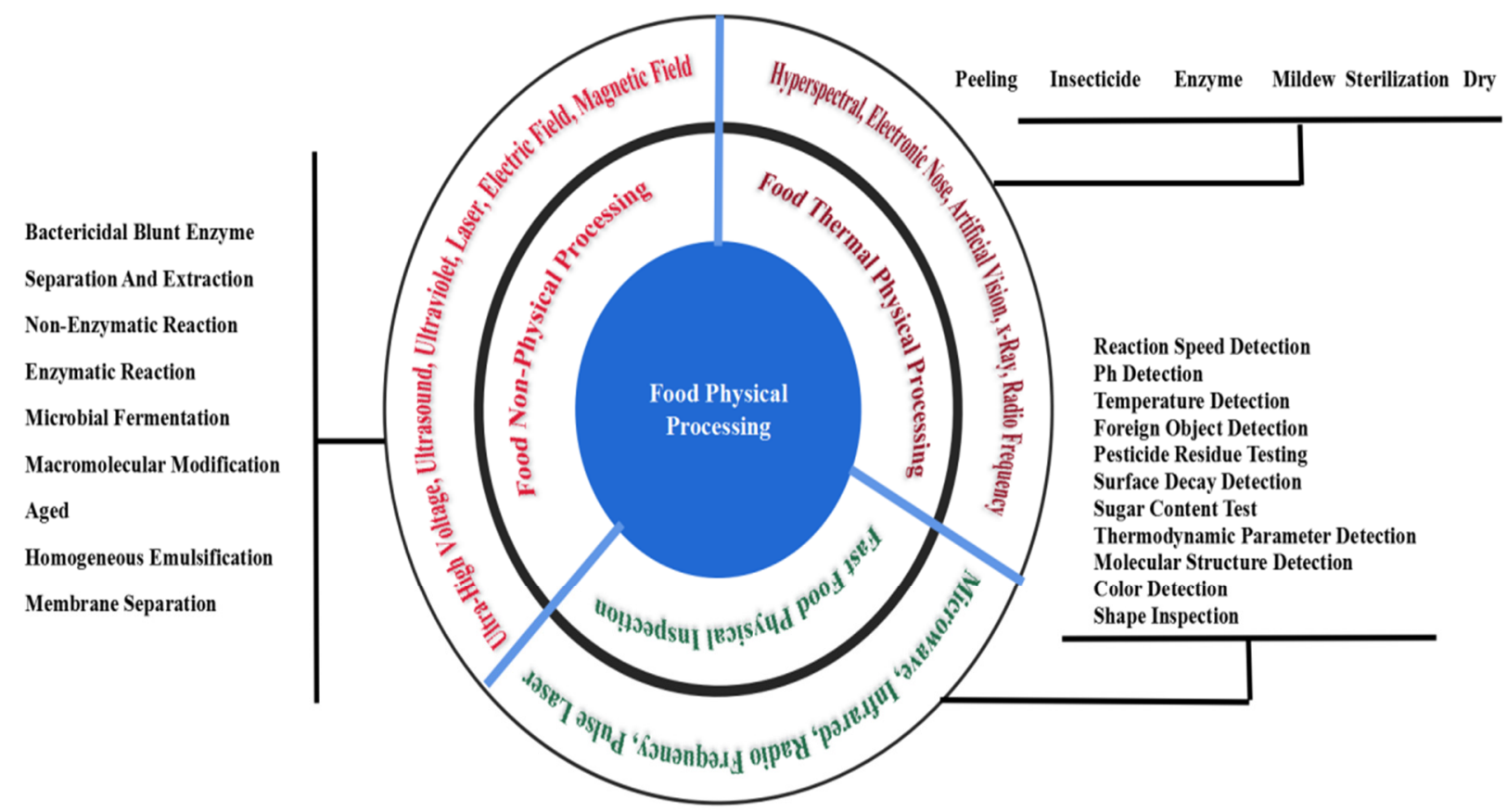

Figure 1 Basic Framework of Food Physical Processing Technology 


\subsection{Food Processing Methods}

It can be seen from the actual research results that due to the differences in food processing methods, the actual food physical processing technology mainly involves traditional food chemical processing, traditional food biological processing and physical processing, etc., as shown in Figure 2. Through this framework, not only can the actual situation of the physical field be displayed, but also promote the wider application of this type of technology in the field of food processing. And its actual value is extremely obvious in the actual extraction, drying and other measures. For example, people can apply ultrasound to fields such as accelerated food infiltration dehydration and hot air drying to enhance membrane separation efficiency.

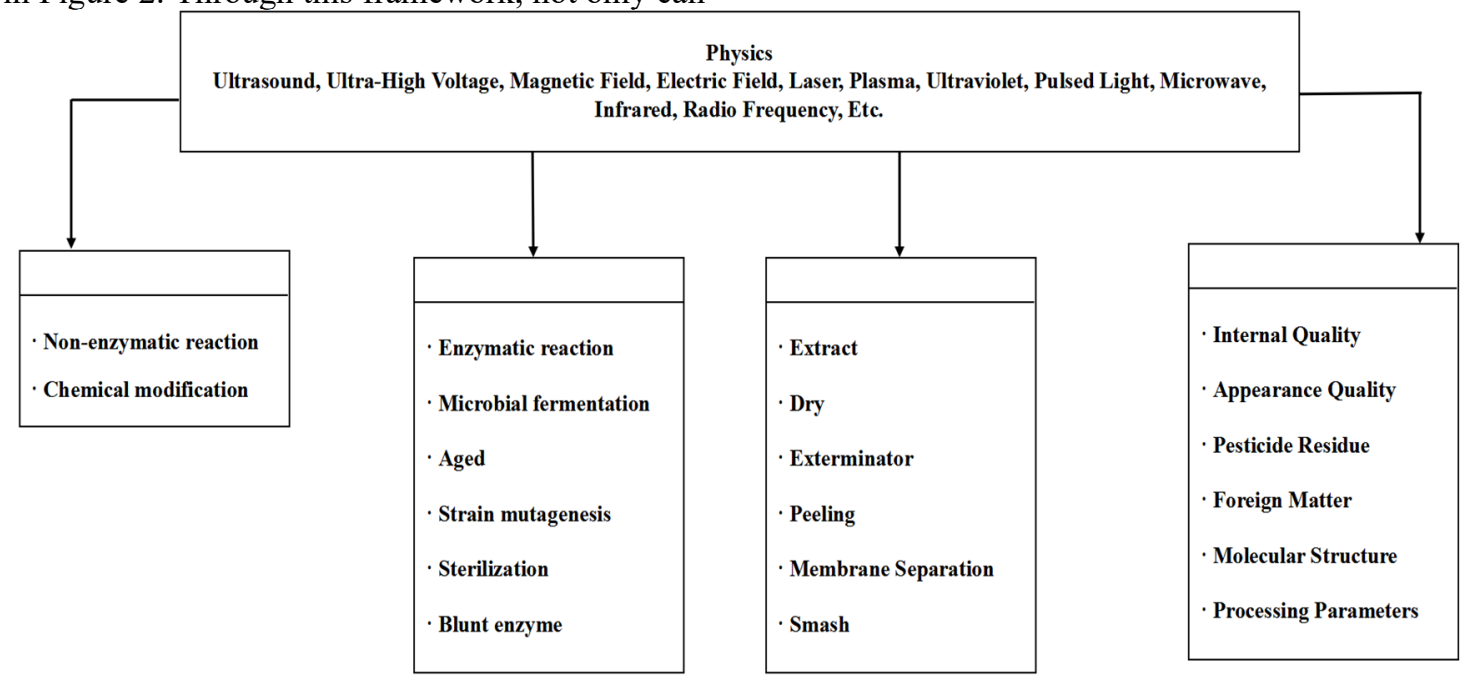

Figure 2 Basic Framework of Food Physical Processing Technology

It can be seen from the construction and application of the first framework that the main focus is on the application advantages of the same physics in different food processing, and the research on the physics is more in-depth. The second framework construction can show the advantages of different physical fields of the same food processing problem. At this time, the common mechanism of different physical fields can also be steadily improved.

\section{Advantages and Characteristics of Food Physical Processing Technology}

\subsection{Efficient and Green}

As can be seen from the implementation of traditional food processing and manufacturing technologies, mainly based on chemistry and biology, after undergoing long-term technical optimization, the room for improvement in processing efficiency has become very limited. Besides, it can be seen from the actual research process that after the introduction of modern physical technology for urinary catheters, it can further improve the product acquisition rate and activity in food processing, and ensure that the extraction, reaction and other links are optimized, which shortens the production time. At the same time, the product production efficiency is greatly improved. In addition, with the continuous application of physical processing methods, not only can the reaction rate be increased, but also the amount of chemical solvents and biocatalysts can be reduced, which is also a basic process for improving the safety of the entire food. In addition, staff can also reduce the processing temperature to avoid the destruction of nutrients in food and enhance their nutritional value.

\subsection{Environmental Protection and Intelligence}

It can be seen from international studies that many researchers use the physical fields of infrared and radio frequency to perform the greening operation of dried fruits and vegetables. This method can avoid the problems of nutrient loss and large water consumption. This is also unmatched by many traditional methods. The use of thermal physics to perform sterilization and insecticide operations can replace traditional sulfur fumigation technology and reduce sulfur dioxide emissions, which is also the fundamental performance of ozone layer protection. There are still many people who use infrared to peel fruits and vegetables to avoid water pollution and other problems caused by the peeling of traditional lye. It can also be seen from this that this method can show good environmental protection effects. At this stage, my country is experiencing the "Industry 4.0" era, and automation and networking have become the goals pursued by the food manufacturing industry. Throughout the entire intelligent manufacturing of food, the main technical bottleneck lies in the rapid collection of process data, which is impossible to achieve with traditional technology, and requires rapid detection by means of infrared and ultrasonic waves. It can also be seen from the above discussion that the physical rapid detection method can play an irreplaceable role in the subsequent intelligent food manufacturing process. Therefore, people should strengthen the research on food 
physical processing technology and its framework, so that food processing is always in good condition.

\section{Conclusion}

In summary, with the rise of food physical processing technology, the development of the food manufacturing industry has become more and more perfect. And because of the in-depth implementation of basic theoretical research work, various new tests and instruments will also be applied, which makes the scientific and technological level of the food processing industry higher and higher. In order to better achieve technological innovation in the food industry, relevant departments and staff need to build a modern food industry system and guide the entire industry to develop in a better direction.

\section{References}

1. Zhou Liyuan, Tang Xiaozhen, Li Ningyang. Application of physical modification technology in comprehensive utilization of food processing by-products [J]. Chinese Condiments, 2019, 44(10): $178-181+186$.

2. Duan Yuqing, He Ronghai, Ma Haile. Frontier teaching practice of food physical processing technology[J]. Agricultural Engineering, 2019, 9(09): 113-115.

3. Liu Mengjia. A summary of the wastewater treatment technology in the food industry [J]. Architecture and Budget, 2019(04): 32-36.

4. Sun Kailian, Li Yunfu. Exploration and practice of physical chemistry teaching in food specialty[J]. Guangzhou Chemical Industry, 2019, 47(21): 158-159+167.

5. Ma Haile. Food physical processing technology and its basic framework [J]. Chinese Journal of Food Science, 2018, 18(04): 1-7. 